\title{
AVALIAÇÃO DOS RESULTADOS DA SINOVECTOMIA POR VIA ARTROSCÓPICA DO COTOVELO EM PACIENTES PORTADORES DE ARTRITE REUMATOIDE
}

\author{
ASSESSMENT OF ARTHROSCOPIC ELBOW SYNOVECTOMY \\ OUTCOMES IN PATIENTS WITH RHEUMATOID ARTHRITIS
}

\begin{abstract}
Alberto Naoki Miyazaki', Marcelo Fregoneze 2 , Pedro Doneux Santos ${ }^{3}$, Luciana Andrade da Silva ${ }^{4}$, Rodrigo Tormin Ortiz ${ }^{5}$, Eduardo César Moreira Mariz Pinto ${ }^{5}$, Sergio Luis Checchia'
\end{abstract}

\section{RESUMO}

Objetivo: Rever os resultados funcionais da sinovectomia por via artroscópica de cotovelo em pacientes com artrite reumatoide. Métodos: Entre maio de 1999 e dezembro de 2005, 15 pacientes foram submetidos à sinovectomia do cotovelo pela técnica artroscópica. Três casos eram bilaterais, totalizando 18 cotovelos. Dois pacientes eram do sexo masculino e 13 do feminino. A média da idade era de 44 anos e cinco meses. O tempo médio de diagnóstico prévio da doença foi de seis anos e oito meses. Todos os pacientes tinham dor no período pré-operatório e em sete cotovelos havia instabilidade. A média das mobilidades articulares no período préoperatório foi: flexão de $118^{\circ}$, extensão de $-24^{\circ}$, supinação de $80^{\circ}$ e pronação de $71^{\circ}$. Resultado: O seguimento pós-operatório médio foi de 39 meses. A média da mobilidade articular pós-operatória de flexão foi de $133^{\circ}$, extensão de $-20^{\circ}$, supinação de $84^{\circ}$ e pronação de $78^{\circ}$. Em nove cotovelos (50\%) houve melhora do arco de movimento pós-operatório para amplitude de movimento funcional. Doze casos $(66,6 \%)$ apresentaram resolução ou melhora da dor para um nível que não interferisse nas atividades de vida diária. Pelo método de avaliação de Bruce, os resultados foram: sete excelentes, três bons, dois regulares e seis ruins, com média de 85,5 pontos. Ocorreu recidiva da sinovite em seis casos $(33,3 \%)$ e a progressão para osteoartrose em quatro (22,2\%). Conclusão: A sinovectomia por via artroscópica do cotovelo em pacientes com artrite reumatoide propicia melhora da dor em $66,6 \%$ dos casos, porém, não leva a melhora significativa do arco de movimento.

Descritores - Artrite reumatoide; Cotovelo; Artroscopia

\section{ABSTRACT}

Objective: To review functional outcomes of arthroscopic elbow synovectomy in patients with rheumatoid arthritis. Methods: Between May 1999 and December 2005, 15 patients were submitted to elbow synovectomy using an arthroscopic approach. Three cases were bilateral, totaling 18 elbows. There were two male and 13 female patients. The mean age was 44 years and five months. The mean time of previous diagnosis was six years and eight months. All patients reported preoperative pain, and on seven elbows, instability was present. The mean preoperative values for joint motion were: flexion, $118^{\circ}$; extension, $-24^{\circ}$, supine, $80^{\circ}$, and; prone, $71^{\circ}$. Result: The mean postoperative follow-up time was 39 months. The mean postoperative joint motion was $133^{\circ}$ for flexion, $-20^{\circ}$ for extension, $84^{\circ}$ supine, and $78^{\circ}$ prone. On nine elbows (50\%) an improved postoperative range of motion was reported, reaching functional levels. Twelve cases (66.6\%) showed pain resolution or improvement to a level not interfering on the activities of daily life. According to Bruce's assessment method, the results were as follows: seven excellent, three good, two fair and six poor results, with an average of 85.5 points. Synovitis recurrence was found in six cases $(33.3 \%)$, and evolution to osteoarthrosis was found in four (22.2\%). Conclusion: Arthroscopic elbow synovectomy in patients with rheumatoid arthritis leads to pain improvement in $66.6 \%$ of the cases; however, it does not cause a significant range of motion improvement.

Keywords - Arthritis, Rheumatoid; Elbow; Arthroscopy

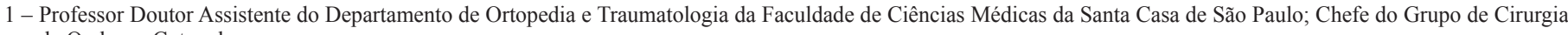
de Ombro e Cotovelo.

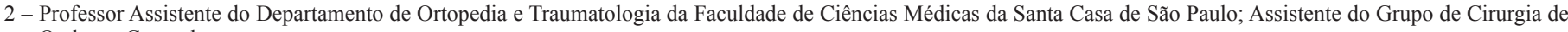
Ombro e Cotovelo.

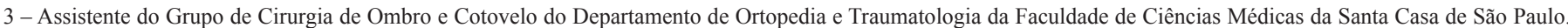

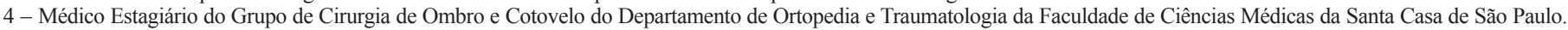

5 - Professor Doutor Adjunto; Chefe de Clínica do Departamento de Ortopedia e Traumatologia da Faculdade de Ciências Médicas da Santa Casa de São Paulo.

Trabalho realizado no Departamento de Ortopedia e Traumatologia da Faculdade de Ciências Médicas da Santa Casa de São Paulo, "Pavilhão Fernandinho Simonsen. Diretor: Professor Doutor Osmar Avanzi.

Correspondência: Santa Casa de Misericórdia de São Paulo, Departamento de Ortopedia e Traumatologia, "Pavilhão Fernandinho Simonsen",

Rua Dr. Cesário Mota Júnior, 112 - 01220-020 - São Paulo, SP. E-mail: ombro@ombro.med.br. Site: www.ombro.med.br

Declaramos inexistência de conflito de interesses neste artigo 


\section{INTRODUÇÃO}

A artrite reumatoide (AR) é uma doença inflamatória, sistêmica e crônica do tecido conjuntivo; sua etiologia é desconhecida e afeta em torno de $0,3 \%$ a $1 \%$ da população mundial. A principal característica da AR é o acometimento articular simétrico e o processo inflamatório é gradualmente crescente $^{(1)}$. Em pacientes portadores de AR com cinco anos de evolução da doença, o acometimento da articulação do cotovelo ocorre em torno de $20 \%$ a $50 \%$ destes ${ }^{(1,2)}$.

Inicialmente, o tratamento conservador consiste em medidas para aliviar a dor e preservar a amplitude de movimento da articulação. A progressão da doença resulta em piora dos sintomas e instabilidade $\operatorname{articular}^{(3)}$, com dor e limitação do cotovelo, que acabam comprometendo a função do membro superior ${ }^{(4)}$.

A sinovectomia cirúrgica está indicada nos casos de falha do tratamento conservador. Quando realizada por via aberta, leva a bons resultados quanto ao alivio dos sintomas, porém, a dor no período pós-operatório, os riscos de deiscência da ferida e de infecção retardam o início da reabilitação $0^{(5)}$.

A artroscopia de cotovelo é um procedimento relativamente novo e tem algumas indicações, dentre elas, a sinovectomia em pacientes com $\mathrm{AR}^{(6)}$. A sinovectomia por via artroscópica é menos invasiva e permite imediata reabilitação; entretanto, apresenta riscos significativos de lesões neurovasculares ${ }^{(5,7)}$. Vários autores têm mostrado resultados satisfatórios com a sinovectomia por via artroscópica, tanto para alívio da dor quanto para melhora funcional, especialmente nos estágios iniciais da doença ${ }^{(2,5,8)}$.
O objetivo deste trabalho é mostrar os resultados funcionais da sinovectomia por via artroscópica do cotovelo nos pacientes com AR.

\section{MÉTODOS}

Foram avaliados os pacientes com diagnóstico de AR operados pelo Grupo de Ombro e Cotovelo do Departamento de Ortopedia e Traumatologia da Faculdade de Ciências Médicas da Santa Casa de São Paulo - "Pavilhão Fernandinho Simonsen", com acometimento da articulação do cotovelo, submetidos à sinovectomia pela técnica artroscópica.

No período de maio de 1999 a dezembro de 2005, 15 pacientes foram submetidos ao tratamento, sendo três com acometimento bilateral, totalizando 18 cotovelos.

Treze pacientes eram do sexo feminino e dois do masculino. A média da idade foi de 44 anos e cinco meses, variando de 16 a 64 anos. Em nove casos (50\%) houve acometimento do membro superior dominante. Dois pacientes $(11 \%)$ apresentavam monoartrite do cotovelo, sem envolvimento de outras articulações (Tabela 1).

O tempo médio de diagnóstico prévio da doença foi de seis anos e oito meses, variando de zero a 23 anos; em três casos a doença foi diagnosticada somente após o procedimento cirúrgico, o qual foi indicado por sinovites monoarticulares inespecíficas. Treze pacientes (72\%) faziam uso de medicações antirreumáticas. Em todos os casos a doença estava em atividade. Todos os pacientes tinham queixa de dor no período pré-operatório. Em sete (39\%) havia instabilidade do cotovelo e em 11 (61\%) a articulação era estável (Tabela 1).

Tabela 1 - Dados dos pacientes

\begin{tabular}{|c|c|c|c|c|c|c|c|c|c|c|c|c|c|c|c|c|c|c|}
\hline & NOME & S & ID & DOM & $\mathbf{L}$ & T. DÇA & MONO & MED & CLAS & INS & MOB. PRÉ & PROC & MOB. PÓS & BRUCE & SAT & REC & SEG & PTC \\
\hline & & & (anos) & & & (anos) & & & MAYO & & (FL/EX)(SU/PR) & & (FL/EX)(SU/PR) & & & & (meses) & \\
\hline \multirow[t]{2}{*}{1} & JAFF & M & 38 & + & D & 5 & & + & IV & + & $(135 /-30)(60 / 30)$ & & $(130 /-40)(40 / 30)$ & 52 & & + & 86 & + \\
\hline & & & & & E & 9 & & & II & & $(120 / 0)(80 / 70)$ & & $(130 /-20)(90 / 70)$ & 94 & + & & 56 & \\
\hline \multirow[t]{2}{*}{2} & GMB & $\mathrm{F}$ & 21 & + & D & 2 & & & 1 & & $(130 /-30)(90 / 80)$ & & $(140 /-20)(90 / 80)$ & 96,1 & + & & 91 & \\
\hline & & & & & E & 5 & & + & 1 & & $(40 /-10)(90 / 80)$ & & $(140 /-30)(90 / 80)$ & 94,1 & + & & 52 & \\
\hline \multirow[t]{2}{*}{3} & CCR & $\mathrm{F}$ & 36 & + & $D$ & 1 & & + & 1 & & $(130 / 0)(90 / 90)$ & & $(140 / 0)(90 / 90)$ & 100 & + & & 35 & \\
\hline & & & & & E & 1 & & + & III & & $(120 /-40)(90 / 90)$ & & $(130 /-10)(90 / 90)$ & 96,2 & + & & 36 & \\
\hline 4 & JSA & $\mathrm{F}$ & 16 & & & DIAG CIR & & & 1 & & $(100 /-30)(90 / 80)$ & & $(90 /-30)(90 / 80)$ & 71,4 & & + & 14 & \\
\hline 5 & CAF & $\mathrm{M}$ & 53 & + & & 12 & & + & III & & $(140 /-20)(90 / 90)$ & & $(140 /-60)(70 / 40)$ & 66,7 & + & & 57 & + \\
\hline 6 & VS & $\mathrm{F}$ & 30 & + & & 6 & & + & II & & $(90 /-45)(15 / 10)$ & CAB.RÁD & $(130 / 0)(90 / 80)$ & 86,9 & + & & 25 & \\
\hline 7 & LSP & $\mathrm{F}$ & 52 & & & 23 & & + & II & & $(130 /-40)(50 / 15)$ & & $(140 /-40)(70 / 70)$ & 62,4 & + & + & 73 & + \\
\hline 8 & $\mathrm{MHL}$ & $\mathrm{F}$ & 64 & & & 10 & & + & IV & + & $(130 / 0)(90 / 80)$ & & $(150 /-60)(90 / 90)$ & 73,1 & & + & 21 & + \\
\hline 9 & $\mathrm{CAE}$ & $\mathrm{F}$ & 56 & + & & 10 & & + & III & + & $(120 /-40)(90 / 50)$ & & $(130 /-10)(90 / 70)$ & 92,5 & + & & 18 & \\
\hline 10 & TRGS & $\mathrm{F}$ & 57 & & & DIAG CIR & + & & III & + & $(120 /-20)(90 / 90)$ & & $(130 /-10)(90 / 90)$ & 81,2 & & + & 17 & + \\
\hline 11 & OAMN & $\mathrm{F}$ & 64 & & & 15 & & + & III & + & $(120 /-30)(90 / 90)$ & & $(130 /-15)(90 / 90)$ & 97,2 & + & & 18 & \\
\hline 12 & NLC & $\mathrm{F}$ & 55 & + & & 10 & & + & III & + & $(110 /-40)(80 / 80)$ & & $(130 /-10)(90 / 90)$ & 98 & + & & 24 & \\
\hline 13 & SMP & $\mathrm{F}$ & 54 & + & & 8 & & + & III & + & $(140 / 0)(80 / 80)$ & & $(140 / 0)(80 / 80)$ & 80 & & + & 18 & + \\
\hline 14 & AMA & $\mathrm{F}$ & 30 & + & & DIAG CIR & + & & 1 & & $(110 /-40)(90 / 90)$ & & $(140 / 0)(90 / 90)$ & 100 & + & & 36 & \\
\hline 15 & EJS & $\mathrm{F}$ & 40 & & & 4 & & + & III & & $(140 /-20)(90 / 90)$ & & $(140 /-10)(90 / 90)$ & 98 & + & & 28 & \\
\hline
\end{tabular}

LEGENDA: $S=\operatorname{sexo}, M=$ masculino, $F=$ feminino, ID = idade, $D O M=$ dominância, $L=$ lado, $D=$ direito, $E=$ esquerdo, $T$. $D C A=$ tempo de doença, DIAG CIR = diagnóstico após a cirurgia

$\mathrm{MONO}=$ envolvimento monoarticular, MED = medicação antirreumatóide, $\mathrm{CLAS}=$ classificação, INST $=$ instabilidade, MOB. $\mathrm{PRE}=$ mobilidade pré-operatória, $\mathrm{FL}=$ flexão, $E X=$ extensão, $S \mathrm{SU}=$ supinação,

$\mathrm{PR}=$ pronação, $\mathrm{PROC}=$ procedimento associado, $\mathrm{CAB}$. RÁDIO = ressecção da cabeça do rádio, MOB. PÓS = mobilidade pós-operatória, $S A T=$ satisfação, $R E C=$ recidiva, $S E G=$ seguimento,

PTC = indicada artroplastia total de cotovelo 
A flexão média no período pré-operatório foi de $118^{\circ}$, variando de $40^{\circ}$ a $140^{\circ}$. A extensão média foi de $-24^{\circ}$, com variação de $0^{\circ}$ a $-45^{\circ}$. A supinação variou de $15^{\circ}$ a $90^{\circ}$, com média de $80^{\circ}$, e a pronação foi em média de $71^{\circ}$, variando de $10^{\circ}$ a $90^{\circ}$ (Tabelas 1 e 2 ).

Tabela 2 - Mobilidade média do cotovelo

\begin{tabular}{l|c|c|c}
\hline & Pré-OP & Pós-OP & Diferença \\
\hline Flexão & $118^{\circ}$ & $133^{\circ}$ & $>15^{\circ}$ \\
\hline Extensão & $-24^{\circ}$ & $-20^{\circ}$ & $>4^{\circ}$ \\
\hline Supinação & $80^{\circ}$ & $84^{\circ}$ & $>4^{\circ}$ \\
\hline Pronação & $71^{\circ}$ & $78^{\circ}$ & $>4^{\circ}$ \\
\hline
\end{tabular}

FONTE: Arquivo DOT-FCMSCSP

Os pacientes foram submetidos a radiografias pré-operatórias em anteroposterior e perfil (Figuras 1 e 2) e exame físico, para enquadramento, segundo a classificação de Mayo, para o comprometimento do cotovelo na artrite reumatoide $^{(9)}$ (Quadro 1), sendo cinco (27\%) considerados como grau I, três como grau II (16\%), oito como grau III (44\%) e dois como grau IV (11\%) (Tabela 1$)$.

Todos os pacientes foram submetidos à artroscopia do cotovelo em decúbito ventral. Em todos foi possível realizar a sinovectomia e, em um caso, foi associada à ressecção da cabeça do rádio. Não encontramos complicações neurovasculares.
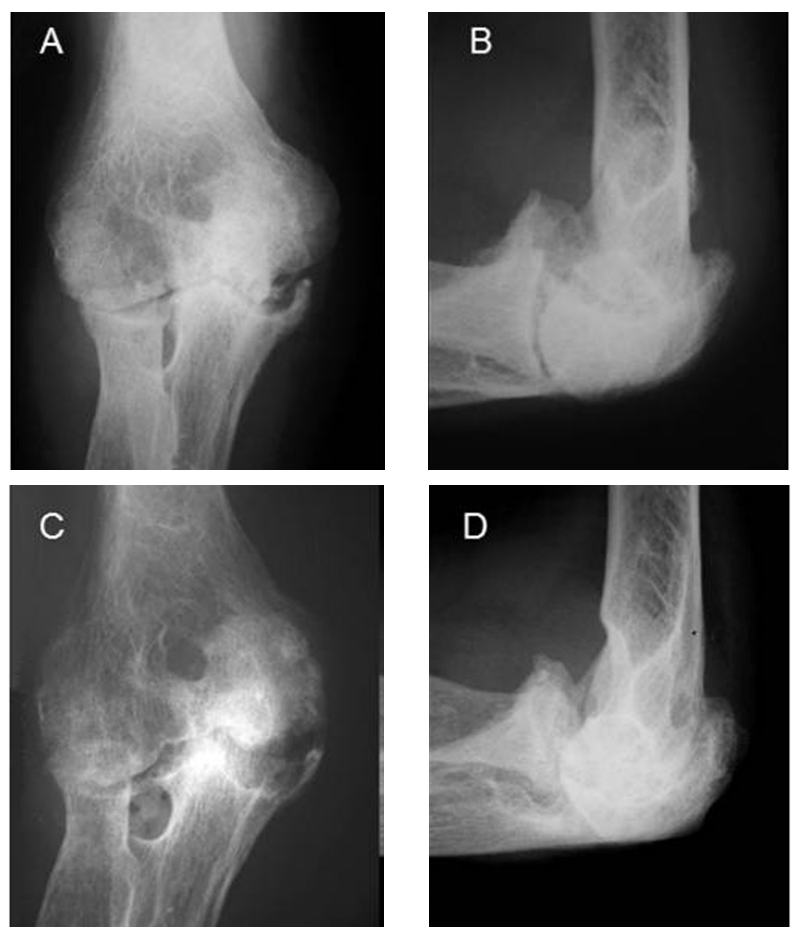

Figura 1 - Radiografias do cotovelo direito do caso 1, classificado com grau IV: A) AP pré-operatório; B) perfil pré-operatório; C) AP pós-operatório; D) perfil pós-operatório.
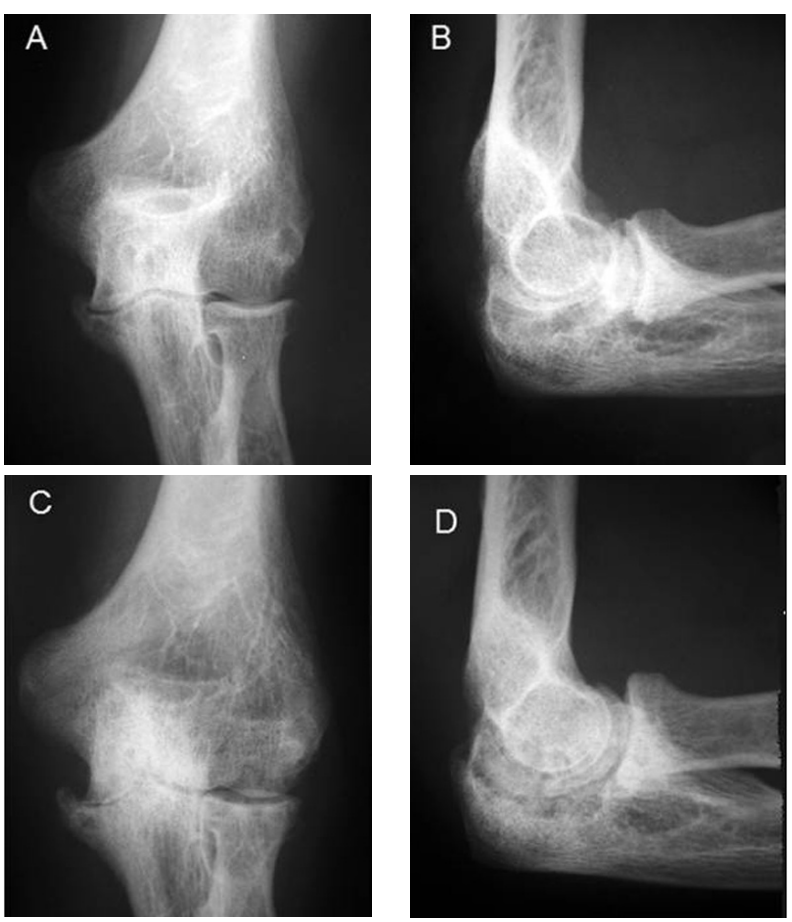

Figura 2 - Radiografias do cotovelo esquerdo do caso 1 classificado como grau II: A) AP pré-operatório; B) perfil pré-operatório; C) AP pósoperatório; D) perfil pós-operatório.

Quadro 1 - Classificação de Mayo para acometimento do cotovelo na artrite reumatoide

\begin{tabular}{|c|l|}
\hline Grau & \multicolumn{1}{|c|}{ Descrição } \\
\hline I & Sem alterações radiográficas além de osteoporose. Presença de sinovite. \\
\hline II & Leve redução do espaço articular. Arquitetura articular preservada. \\
\hline & Persistência da sinovite. \\
\hline III & $\begin{array}{l}\text { Moderadas a graves alteraçães da arquitetura do cotovelo. Afinamento } \\
\text { do olécrano ou reabsorção da tróclea ou capítulo. Sinovite variável } \\
\text { podendo estar quiescente. }\end{array}$ \\
\hline IV & $\begin{array}{l}\text { Grande destruição articular, com reabsorção da superfície articular. } \\
\text { Mínima sinovite. Presença de instabilidade. }\end{array}$ \\
\hline
\end{tabular}

Modificado de Morrey et al. 1992

Quatro pacientes (casos 1, 4, 8 e 10) necessitaram de outros procedimentos cirúrgicos, por via artroscópica ou por via aberta, no acompanhamento pós-operatório; para esses pacientes a data dessa operação foi considerada a de avaliação final.

Com relação aos resultados, os pacientes foram avaliados quanto ao grau de mobilidade articular e submetidos ao questionário pelos critérios da American Medical Association (AMA) modificados por Bruce et al ${ }^{(10)}$ (Quadro 2).

\section{RESULTADOS}

O seguimento pós-operatório foi em média de 39,1 meses, variando de 14 a 91 meses (Tabela 1).

Quanto à mobilidade articular no período pós-operatório, a flexão média foi de $133^{\circ}$, com variação de $90^{\circ}$ a 
Quadro 2 - Critérios de avaliação da AMA modificados por Bruce et al

\begin{tabular}{|c|l|}
\hline \multicolumn{2}{|c|}{ Arco de movimento (ADM) (60 pontos) } \\
\hline \multicolumn{2}{|c|}{ Número de pontos de ADM = 60 - (porcentagem de limitação do membro superior X 0,6) } \\
\hline Capacidade de realização das atividades de vida diária (AVD) (20 pontos) \\
\hline 20 & Função igual à do braço contralateral \\
\hline 15 & AVD independente; menos que duas limitações de trabalho \\
\hline 10 & $\begin{array}{l}\text { Incapaz de realizar mais de três AVD; três ou mais limitações de trabalho; } \\
\text { mudança ocupacional necessária }\end{array}$ \\
\hline 5 & Incapaz de realizar mais de quatro AVD; incapacidade ocupacional \\
\hline Dor (15 pontos) \\
\hline 15 & Sem dor \\
\hline 13 & Dor leve sem comprometimento das atividades \\
\hline 10 & Dor que interfere nas atividades \\
\hline 5 & Dor impedindo algumas atividades \\
\hline 0 & Dor causando impossibilidade e impedindo algumas atividades \\
\hline Anatomia (5 pontos) \\
\hline 1 & Aparência cosmética aceitável \\
\hline 1 & Sem angulação clínica \\
\hline 1 & Sem desvio clínico \\
\hline 1 & Mudança clínica do ângulo de carregamento menor do que 10 \\
\hline 1 & Consolidação clínica \\
\hline Resultados \\
\hline Excelente & 96 - 100 \\
\hline Bom & 91 - 95 \\
\hline Regular & 81 - 90 \\
\hline Ruim & Abaixo de 80 \\
\hline Legenda: AMA = American Medical Association; ADM = arco de movimento; \\
AVD = atividades de vida diária \\
Modificado de Bruce et al. 1974 \\
\hline
\end{tabular}

$150^{\circ}$. A extensão variou de $0^{\circ}$ a $-60^{\circ}$, com média de $-20^{\circ}$. A supinação foi em média de $84^{\circ}$, variando de $40^{\circ}$ a $90^{\circ}$. A média da pronação final foi de $78^{\circ}$, variando de $30^{\circ}$ a $90^{\circ}$. Com relação às mobilidades articulares do período pré-operatório, a flexão aumentou $15^{\circ}$, a extensão $4^{\circ}$, a supinação $4^{\circ}$ e a pronação $7^{\circ}$ (Tabela 2 ).

Em nove cotovelos (50\%) o arco de movimento pósoperatório melhorou para amplitude funcional, ou seja, com no mínimo $130^{\circ}$ de flexão e $-30^{\circ}$ de extensão (Figura 3), conforme descrição de Morrey et $a l^{(11)}$. Os dois casos $(11 \%)$ que apresentavam limitação da supinação e da pronação abaixo da angulação funcional de $50^{\circ} \mathrm{em}$ cada movimento, casos 6 e 7, evoluíram com melhora no período pós-operatório (Tabela 1 ).

Após a cirurgia, em 12 casos $(66,6 \%)$ houve resolução ou melhora da dor a ponto que não interferisse nas atividades da vida diária.

Com relação à capacidade de realização de atividades de vida diária no período pós-operatório, dez cotovelos $(55,5 \%)$ tinham função normal e dois $(11,1 \%)$, atividade de vida diária independente com menos de duas limitações para o trabalho (Quadro 2). Esses pacientes representam $66,6 \%$ do total.

Pelos critérios de avaliação de Bruce, o valor médio encontrado foi de 85,5 pontos. Os resultados foram considerados excelentes em sete pacientes (38\%), bons em três (16\%), regulares em dois (11\%) e ruins em seis (33\%) (Tabela 1$)$.

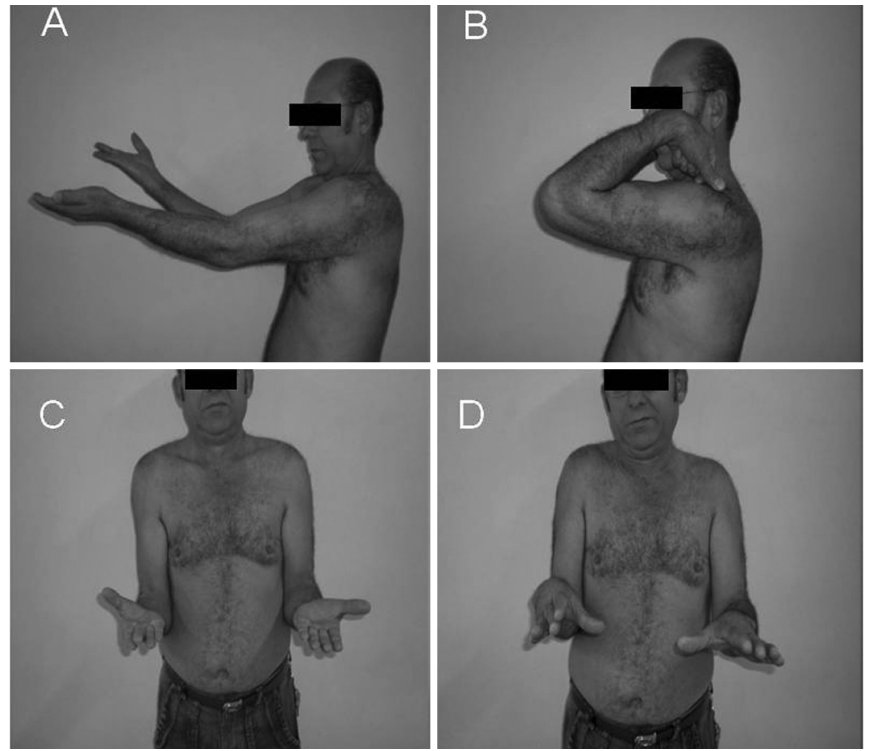

Figura 3 - Imagens do caso 1 com acometimento bilateral em estágios diferentes. Lado direito classificado como grau IV e lado esquerdo como grau II: A) extensão máxima; B) flexão máxima; C) supinação; D) pronação.

\section{DISCUSSÃO}

A realização da sinovectomia no cotovelo de pacientes com AR é indicada na sinovite não controlada por medicação, associada à persistência da dor, rigidez e perda de função ${ }^{(12)}$.

Os resultados da sinovectomia por via aberta do cotovelo em pacientes com AR foram descritos por vários autores, com melhora da dor e amplitude de movimento, porém, o procedimento não é isento de complicações como infecção, deiscência da ferida operatória e fratura do olécrano ${ }^{(4,13-16)}$.

A sinovectomia por via artroscópica é descrita como uma alternativa menos invasiva e com recuperação pósoperatória mais rápida, mas é um procedimento tecnicamente mais difícil, com maior possibilidade de complicações neurovasculares ${ }^{(6,7,12)}$. Não observamos essas complicações no nosso estudo.

Horiuchi et $a l^{(1)}$ acreditam que a sinovectomia por via artroscópica do cotovelo na AR não tenha como principal objetivo o ganho de arco de movimento, mas sim o alívio da dor e, secundariamente, melhora para realização de atividades diárias. Vários autores também relatam que não ocorre melhora substancial da mobilidade articular no período pós-operatório ${ }^{(2,5,12)}$, o que coincidiu com as nossas observações. Houve alguma melhora na mobilidade articular em todas as direções do movimento; o principal incremento ocorreu na flexão, com aumento médio de $15^{\circ}$ (Tabela 2).

Com relação à melhora da dor, 12 cotovelos $(66,6 \%)$ evoluíram sem dor ou com dor leve, que não interferia nas atividades diárias. Resultados semelhantes apresentaram Lee e Morrey ${ }^{(2)}$, com 64,2\% dos pacientes sem dor ou 
com dor leve após a realização da sinovectomia por via artroscópica. Horiuchi et al ${ }^{(1)}$ tiveram $76 \%$ dos pacientes em situação semelhante. Tanaka et al ${ }^{(5)}$ compararam pacientes com AR submetidos à sinovectomia do cotovelo tanto por via aberta como por via artroscópica; $48 \%$ dos submetidos à cirurgia por artroscopia e $70 \%$ por via aberta não apresentaram dor ou tiveram dor leve, porém, tal diferença não foi estatisticamente significativa.

A ressecção da cabeça do rádio é descrita por autores que realizam a cirurgia por via aberta ${ }^{(4,14,16)}$. Porém, Copeland e Taylor ${ }^{(15)}$ mostram que é possível alcançar bons resultados apenas com a sinovectomia do cotovelo, sem ressecção da cabeça do rádio, pois ela atua como estabilizadora do cotovelo e do punho. Portanto, é desejável mantê-la no cotovelo reumatoide; a ressecção está contraindicada em casos com instabilidade ${ }^{(3)}$. No nosso estudo, a ressecção da cabeça do rádio foi feita em apenas um paciente (caso 6), que apresentava limitação da mobilidade articular e dor principalmente com relação à supinação e pronação. $\mathrm{Na}$ avaliação pós-operatória esse caso apresentou melhora desses movimentos.

Segundo Kauffman et al $l^{(3)}$, o grau de acometimento do cotovelo no período pré-operatório é descrito como importante fator prognóstico; eles mostraram que, mesmo em casos com comprometimento significativo da articulação, a sinovectomia e a liberação capsular podem resultar em melhora da dor e do arco de movimento. Pudemos observar que os pacientes que apresentaram piores resultados eram aqueles com graus mais elevados na classificação pré-operatória (Mayo) (Tabela 1).

Os resultados ruins foram atribuídos à recidiva da sinovite em seis casos (33\%) e a progressão da osteoartrose em quatro deles $(22 \%)$.

Em três pacientes (16\%) (casos 1, 8 e 10), a evolução da artrite dos cotovelos levou à realização da artroplastia total. Em outros três casos (16\%) (pacientes 5, 7 e 13), a artroplastia total foi indicada, mas ainda não foi realizada (Tabela 1 ).

\section{REFERÊNCIAS}

1. Horiuchi K, Momohara S, Tomatsu T, Inoue K, Toyama Y. Arthroscopic synovectomy of the elbow in rheumatoid arthritis. J Bone Joint Surg Am. 2002;84(3):342-7.

2. Lee BP, Morrey BF. Arthroscopic synovectomy of the elbow for rheumatoid arthritis. J Bone Joint Surg Br.1997;79(5):770-2.

3. Kauffman JI, Chen AL, Stuchin S, Di Cesare PE. Surgical management of the rheumatoid elbow. J Am Acad Orthop Surg. 2003;11(2):100-8.

4. Brumfield RH Jr, Resnick CT. Synovectomy of the elbow in rheumatoid arthritis. J Bone Joint Surg Am. 1985;67(1):16-20.

5. Tanaka N, Sakahashi H, Hirose K, Ishima T, Ishii S. Arthroscopic and open synovectomy of the elbow in rheumatoid arthritis. J Bone Joint Surg Am. 2006;88(3):521-5.

6. O'Driscoll SW, Morrey BF. Arthroscopy of the elbow. J Bone Joint Surg Am. 1992;74(1):84-94

7. O'Driscoll SW. Elbow arthritis: treatment options. J Am Acad Orthop Surg. 1993;1(2):106-16

8. Nemoto K, Arino H, Yoshihara Y, Fujikawa K. Arthroscopic synovectomy for the rheumatoid elbow: a short-term outcome. J Shoulder Elbow Surg. 2004;13(6):352-5.

9. Morrey BF, Adams RA. Semiconstrained arthroplasty for treatment of rheumatoid arthritis of the elbow. J Bone Joint Surg Am.1992;74(4):479-90.
A exceção foi um caso de paciente portadora de artrite reumatoide juvenil diagnosticada na primeira cirurgia (caso 4), que, mesmo com o grau de acometimento do cotovelo classificado com grau I, evoluiu com recidiva dos sintomas, necessitando de uma nova sinovectomia artroscópica após 14 meses. Esse segundo procedimento também não obteve sucesso, o que levou a paciente a ser submetida a uma artroplastia de interposição e à ressecção da cabeça do rádio após 18 meses (Tabela 1).

Dos três pacientes submetidos à cirurgia bilateral, em dois (casos 2 e 3) o grau de acometimento era baixo (grau I de Mayo) e evoluíram sem intercorrências, com satisfação dos pacientes. No paciente que apresentava os cotovelos em graus diferentes de acometimento (caso 1), o lado com grau mais avançado foi submetido à artroplastia total devido à persistência dos sintomas após a sinovectomia (Figuras 1, 2 e 3 ).

O índice de reoperação foi de 22\%: em uma paciente (caso 4), nova sinovectomia foi realizada por via artroscópica e. posteriormente. uma artroplastia de interposição; em outros três pacientes foram realizadas artroplastias totais (casos 1, 8 e 10). Desses, os casos 1 e 8 , classificados inicialmente como grau IV, não tiveram boa evolução, como, eventualmente, era de esperar, e foram submetidos à artroplastia total. O nosso índice de reoperação é similar ao verificado por Mäenpää et $a l^{(17)}$, os quais realizaram 103 sinovectomias por via aberta do cotovelo e reoperaram 22 pacientes $(21 \%)$, sendo oito repetições da sinovectomia e 14 artroplastias totais.

Com relação ao índice de satisfação dos nossos pacientes, $12(66,6 \%)$ ficaram contentes com os resultados dos procedimentos aos quais foram submetidos.

\section{CONCLUSÃO}

A sinovectomia por via artroscópica de cotovelo em pacientes com artrite reumatoide propiciou melhora da dor em $66,6 \%$ dos cotovelos operados, porém, não levou à melhora da mobilidade articular.

10. Bruce HE, Harvey JP Jr, Wilson JC Jr. Monteggia fractures. J Bone Joint Surg Am.1974;56(8):2563-76.

11. Morrey BF, Askew LJ, Chao EY. A biomechanical study of normal functional elbow motion. J Bone Joint Surg Am.1981;63(6):872-7.

12. Steinmann SP, King GJ, Savoie FH 3rd. Arthroscopic treatment of the arthritic elbow. J Bone Joint Surg Am.2005;87(9):2114-21.

13. Inglis AE, Ranawat CS, Strau LR. Synovectomy and débridement of the elbow in rheumatoid arthritis. J Bone Joint Surg Am.1971;53(4):652-62.

14. Porter BB, Richardson C, Vainio K. Rheumatoid arthritis of the elbow: the results of synovectomy. J Bone Joint Surg Am.1974;56(3):427-37.

15. Copeland SA, Taylor JG. Synovectomy of the elbow in rheumatoid arthritis. J Bone Joint Surg Br.1979;61(1):69-73.

16. Eichenblat M, Hass A, Kessler I. Synovectomy of the elbow in rheumatoid arthritis. J Bone Joint Surg Am.1982;64(7):1074-8.

17. Mäenpää HM, Kuusela PP, Kaarela K, Kautiainen HJ, Lehtinen JT, Belt EA. Reoperation rate after elbow synovectomy in rheumatoid arthritis. J Shoulder Elbow Surg. 2003;12(5):480-3. 